\title{
Reconstruction of a large lower lip defect using a combination of Abbe and staircase flaps: a case report
}

\author{
Bo Min Moon, \\ Woo Sik Pae \\ Department of Plastic and \\ Reconstructive Surgery, Gachon \\ University Gil Medical Center, Gachon \\ University College of Medicine, Incheon, \\ Korea
}

\begin{abstract}
Lip defects often occur following wide excision as a surgical treatment for squamous cell carcinoma of the oral cavity. Defects larger than one-half of the lip cannot be closed primarily and require flap surgery. Reconstruction of the oral sphincter function can be achieved by means of a local flap using the like tissue, rather than with a free flap utilizing different tissues. A defect of the lower lip requires reconstruction using different techniques, depending on its size and location. Herein, we present the case of a patient exhibiting a lip defect spanning more than two-thirds of the lower lip, after a wide resection due to squamous cell carcinoma. The defect was reconstructed using an Abbe flap and a staircase flap. Revision was performed after 16 days. The patient's oral competencies were fully restored 3 months postoperatively, and the esthetic results were ideal. Based on our experience, a combination of the Abbe and staircase flaps can produce excellent functional and esthetic outcomes in the reconstruction of a lower lip with a large defect. It can serve as a reliable reconstruction option for defects spanning more than two-thirds of the lower lip, not including the oral commissures.
\end{abstract}

Keywords: Case reports / Lip / Reconstructive surgical procedures / Surgical flaps

\section{INTRODUCTION}

The standard therapy for lower lip squamous cell carcinoma is surgical excision [1]. After wide resection, a large surgical defect often occurs. The goals of perioral reconstruction are esthetic and reestablishment of function. The alignment of the vermilion border is the most important esthetic aspect, as even a small misalignment is noticeable. The lips are essential components for establishing oral competence and facilitating muscular function and mouth opening.

Reconstruction using the same material can facilitate functional recovery. However, the type of reconstruction depends

\section{Correspondence: Woo Sik Pae}

Department of Plastic and Reconstructive Surgery, Gachon University Gil Medical Center, Gachon University College of Medicine, 21 Namdong-daero 774beon-gil,

Namdong-gu, Incheon 21565, Korea

E-mail: 4urdream0153@gmail.com

Received October 5, 2021 / Revised October 14, 2021 / Accepted December 18, 2021 on the remaining healthy lip and location of the defect [2,3]. The use of a good reconstructive approach may be challenging in larger defects. It is generally agreed that reconstruction after resection of more than two-thirds of the lower lip produces poor results [2-4]. For such cases, various methods have been used, and the multiplicity of reported techniques reported indicate that there is no ideal method for reconstructing the entire lower lip.

We executed a combination of the Abbe and staircase flaps to reconstruct more than two-thirds of a lower lip, excluding the commissure area. Studies on this method have been rare [5].

\section{CASE REPORT}

An 81-year-old woman with a history of diabetes and hypertension had a mass in her lower lip that had developed approximately 3 months prior. A punch biopsy was performed at an- 
other hospital, and squamous cell carcinoma was confirmed. The tumor location was central and did not invade the commissure area. The tumor was pedunculated, being $5 \times 4 \times 2 \mathrm{~cm}^{3}$ in dimensions, with a diameter of $4 \mathrm{~cm}$ of the stalk. The total lip width was $6 \mathrm{~cm}$ (Figs. 1, 2). Results of imaging examinations indicated suspected pathologic lymph nodes at levels I and II on both sides of the neck. After consulting with the otorhino-

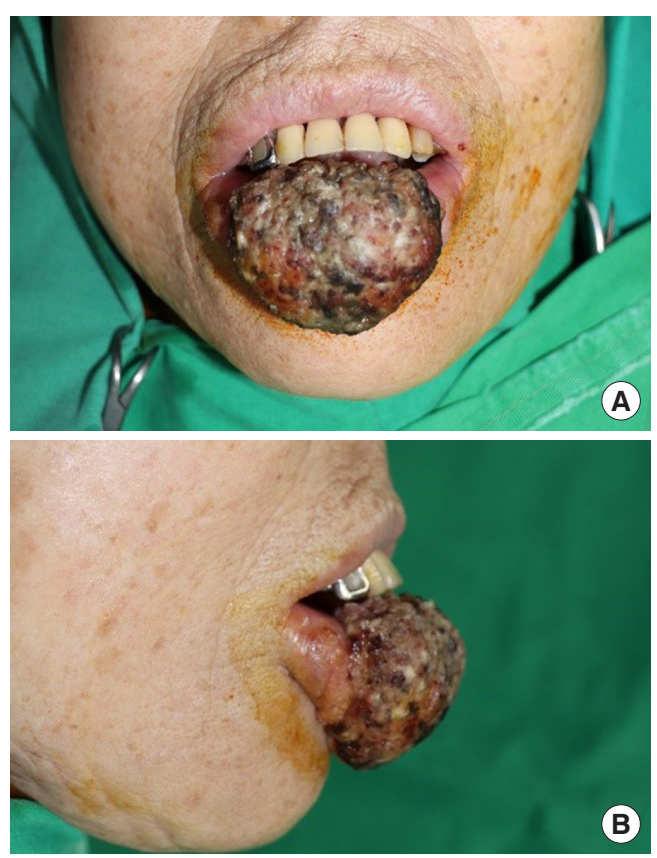

Fig. 1. Preoperative photographs of an 81-year-old woman with a tumor in the lower lip. (A) Anterior view. (B) Lateral view.
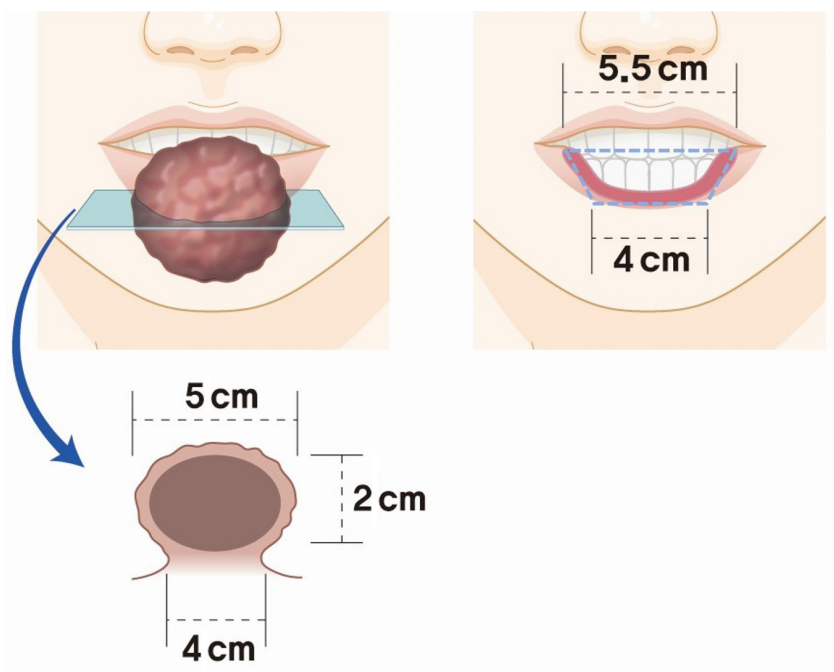

Fig. 2. Schematic diagram of the tumor and defect after wide resection. After the resection, the shape of the lower lip defect is trapezoidal; the upper and lower sides are 5.5 and $4 \mathrm{~cm}$ in length, respectively. The relative size of the defect is more than two-thirds of the total width of the lip. laryngology department, selective neck dissection surgery on both sides was carried out simultaneously.

The surgery was performed under general anesthesia with nasotracheal intubation, followed by a wide excision with a $5-\mathrm{mm}$ safety margin. Tumor-free margins were confirmed through the frozen section pathology examination. After resection, the shape of the lower lip defect was trapezoidal; and the upper and lower sides measured 5.5 and $4 \mathrm{~cm}$, respectively (Fig. 2). The relative defect size was over two-thirds of the total lip width.

In the first stage, the horizontal lengths of the staircase flap were $1.5,1.2,0.9$, and $0.6 \mathrm{~cm}$. Four steps were required to close the defect. The last step was creating a Burow's triangle with the vertex pointing down. The triangle and rectangles formed by the incisions were subsequently resected until exposed, preserving the orbicularis oris muscle layer (Fig. 3A and B).
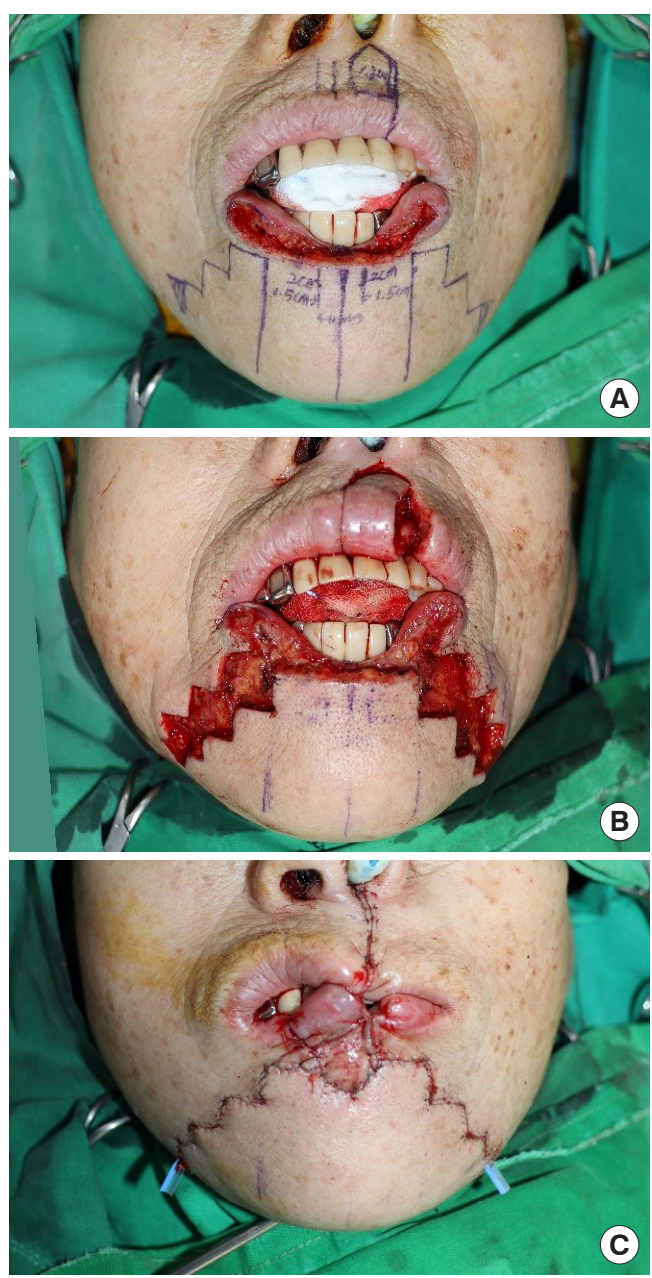

Fig. 3. Intraoperative photographs. (A) A design combines the Abbe flap and staircase flap. (B) The Abbe flap is harvested, and the triangles and rectangles of the staircase flap are excised in the subcutaneous layer while preserving the orbicularis muscle layer. (C) The donor site is closed primarily. Penrose drains are placed at the bottom of each side of the staircase flap. 


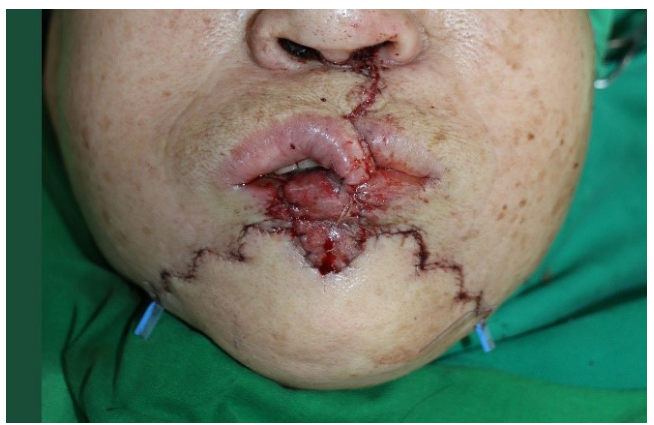

Fig. 4. After making an additional incision along the upper vermilion border to resolve congestion of the Abbe flap within 24 hours postoperatively.

In the second stage, the Abbe flap that is pedicled to the superior labial vessels was designed next to the left philtrum column to avoid damaging the philtrum shape. The flap was pentagonal (width, $1.5 \mathrm{~cm}$ ). The lateral side of the flap was completely divided, and the location of the superior labial artery was confirmed. The medial side of the flap was carefully dissected until the superior labial artery had been identified and preserved. This permitted the easy rotation of the flap onto the central lower lip defect. The upper lip was primarily sutured layer by layer, with careful attention and precision around the pedicle (Fig. 3B).

In the last stage, the staircase flaps were advanced and approximated to the Abbe flap using layered closure. Routine subcutaneous tissue and skin sutures were performed for the excised rectangles and triangles, taking care to place the corner sutures at the step corners (Fig. 3C).

The patient's protruding upper teeth applied tension on the flap, resulting in flap congestion. We revised this by making an additional incision along the upper vermilion border within 24 hours postoperatively (Fig. 4). There was no further congestion or necrosis of the flap. The patient was administered a liquid diet ingested through a straw until the flap stabilized and was divided. After 16 days, the pedicle of the Abbe flap was divided (Fig. 5).

Permanent section pathological examination revealed no tumors on either the salivary glands or the 37 lymph nodes and no lymphovascular or perineural invasion. The patient exhibited relatively normal lip muscle function, normal pronunciation, and no signs of recurrence postoperatively.

\section{DISCUSSION}

Surgical management of oral squamous cell carcinoma typically involves resectioning the carcinoma with a $0.4 \times 0.6 \mathrm{~cm}$ margin of normal-appearing tissue [6]. Large surgical defects are often encountered. Reconstruction methods for the lower lip vary

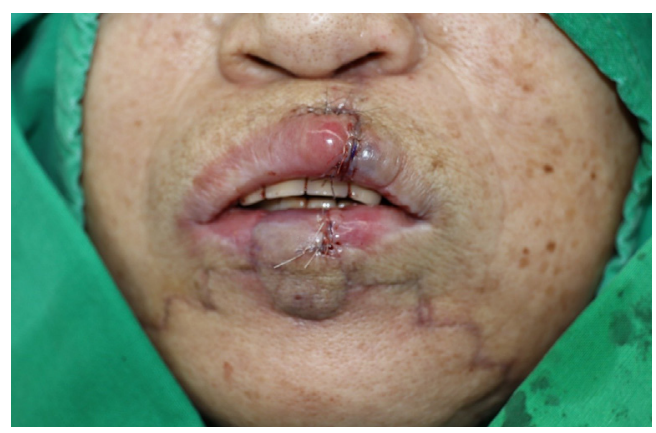

Fig. 5. After division of the Abbe flap pedicle 16 days postoperatively.

depending on the defect size and location. For small defects occupying less than one-third of the lip, reconstruction is possible by performing a "W" or "V" type excision, and suturing it immediately without applying a local flap [2,3]. For defects spanning more than one-third of the lip, tissue from the adjacent area should be used. A rectangular flap is sometimes used for V-shaped resections.

If the defect spans two-thirds of the lip, reconstruction becomes difficult. Various methods such as the Bernard modification (1852) [7], Schuchardt flap (1954) [8], Webster modification (1960) [9], Johanson staircase technique (1974) [10], and Abbe flap (1898) [11] have been reported. The variations suggest that there is no ideal method yet.

The Bernard-Freeman-Fries flap $[7,12,13]$ results in marked scarring, reduced mouth opening, and altered smile kinetics. The Webster flap leads to scarring in the chin area (Schuchardt flap, half-circle scar) due to trapdoor deformity, and is relatively conspicuous in Asian populations [14]. The staircase flap technique results in relatively inconspicuous scarring and prevents this deformity.

The staircase flap, used to close full-thickness defects, is placed using a lateral advancement flap from the lateral parts of the lip and chin in a stepwise fashion [4]. It was first described by Johanson et al. in 1974 [10] and subsequently modified by Kuttenberger and Hardt [15] and Salgarelli et al. [5]. This technique has the advantage of preventing functional and cosmetic sequelae by avoiding transection of the orbicularis oris, depressor labii inferioris, or depressor anguli oris $[2,4]$.

The Abbe flap is a full-thickness, one-lip, switch flap that is pedicled to the superior or inferior labial vessels and rotated $180^{\circ}$ to fit into the defect of the opposite lip. The Abbe and switch flaps were first described by Sabattini (1838) [16], and later by Stein (1848) [17], Estlander (1872) [18], and Abbe (1898) [11]. For defects of the lower lip, it is appropriate to use the tissue next to the philtrum, as the philtrum tissue is not es- 
thetically pleasing. The great advantage of the Abbe flap is that it allows for restoration of the orbicularis oris sphincter as it involves the transfer of full-thickness lip tissue. Neurotization, i.e., redistribution of nerve tissue, has been reported to occur in the muscle region of the Abbe flap [19]. The donor site can be closed primarily, resulting in little scarring. For these reasons, although there is a need for secondary surgery for pedicle division (after 14-28 days), these flaps are widely used.

Our patient had a large defect spanning more than two-thirds of her lower lip. We considered a local flap for functional reconstruction of the oral sphincter and decided to combine two methods to fill the large defect. We performed a combination of the Abbe and staircase flaps due to the advantages mentioned above. The middle third of the lower lip defect was covered with an Abbe flap from the upper lip, and the remaining defects on both sides were covered with the staircase flap.

There have been few studies on combining the two surgical techniques, and there were no data on potential postoperative complications [5]. In this case, we report complications that occurred after reconstruction of the lower lip defect following the removal of a large tumor. Although the Abbe flap was designed for a tension-free closure, postoperative congestion occurred in the flap. We found that the patient's upper teeth were protruded due to a dental bridge that could not be removed (Fig. 1B). When the patient's movement was enabled after recovery from general anesthesia, tension was applied to the Abbe flap, leading to congestion. We relieved the tension by performing pedicle lengthening with an additional incision along the upper vermilion border, within 24 hours postoperatively (Fig. 4). Hence, this report further indicates the necessity of considering the degree of protrusion of the patient's teeth to ensure sufficient pedicle length in the Abbe flap.

Satisfactory results were obtained postoperatively regarding the patient's lip movement, pronunciation, and esthetics. In conclusion, this approach may serve as a reliable reconstruction option for patients with lip cancer, with defects of more than two-thirds of the lower lip, excluding the oral commissures. This method may be used to successfully reconstruct a large defect of the lower lip caused by a variety of factors.

\section{NOTES}

\section{Conflict of interest}

No potential conflict of interest relevant to this article was reported.

\section{Ethical approval}

The study was approved by the Institutional Review Board of Ga- chon University Gil Medical Center (IRB No. GDIRB2021-319).

\section{Patient consent}

The patient provided written informed consent for the publication and the use of her images.

\section{ORCID}

Bo Min Moon

https://orcid.org/0000-0001-6209-096X

Woo Sik Pae

https://orcid.org/0000-0002-4131-7931

\section{Author contribution}

Conceptualization: Woo Sik Pae. Formal analysis: Bo Min Moon. Methodology: Woo Sik Pae. Project administration: Woo Sik Pae. Writing - original draft: Bo Min Moon. Writing review \& editing: Bo Min Moon, Woo Sik Pae. Investigation: Bo Min Moon. Software: Bo Min Moon. Supervision: Woo Sik Pae. Validation: Bo Min Moon, Woo Sik Pae.

\section{REFERENCES}

1. Agostini T, Spinelli G, Arcuri F, Perello R. Metastatic squamous cell carcinoma of the lower lip: analysis of the 5-year survival rate. Arch Craniofac Surg 2017;18:105-11.

2. Kim HI, Kim HS, Park JH, Yi HS, Kim YS, Kim HY. Reconstruction of a small defect of the lower vermilion adjacent to white roll using a modified O-Z flap. Arch Craniofac Surg 2021;22:164-7.

3. Ki SH, Jo GY, Yoon J, Choi MSS. Reconstruction of microstomia considering their functional status. Arch Craniofac Surg 2020;21:161-5.

4. Roldan JC, Schulte-Mattler W. Stein's double cross-lip flaps combined with Johanson's step technique for subtotal lower lip reconstruction. Plast Reconstr Surg Glob Open 2016;4:e615.

5. Salgarelli AC, Bellini P, Magnoni C, Anesi A, Collini M. Synergistic use of local flaps for total lower lip reconstruction. Dermatol Surg 2011;37:1666-70.

6. Khan AA, Potter M, Cubitt JJ, Khoda BJ, Smith J, Wright EH, et al. Guidelines for the excision of cutaneous squamous cell cancers in the United Kingdom: the best cut is the deepest. J Plast Reconstr Aesthet Surg 2013;66:467-71.

7. Bernard C. Cancer of the lower lip: restoration with the help of quadrilateral-lateral flaps. Scalpel 1852;5:162-4.

8. Schuchardt K. Operations in the face and in the jaw area operations on the lips. In: Bier A, Braun H, Kummel H, editors. Surgical operation theory. Leipzig: J.A. Barth; 1954.

9. Webster RC, Coffey RJ, Kelleher RE. Total and partial reconstruction of the lower lip with innervated musclebearing flaps. Plast Reconstr Surg Transplant Bull 1960;25:360-71. 
10. Johanson B, Aspelund E, Breine U, Holmstrom H. Surgical treatment of non-traumatic lower lip lesions with special reference to the step technique: a follow-up on 149 patients. Scand J Plast Reconstr Surg 1974;8:232-40.

11. Abbe R. A new plastic operation for the relief of deformity due to double harelip. Plast Reconstr Surg 1968;42:481-3.

12. Freeman BS. Myoplastic modification of the Bernard cheiloplasty. Plast Reconstr Surg Transplant Bull 1958;21:453-60.

13. Fries R. Advantages of a basic concept in lip reconstruction after tumour resection. J Maxillofac Surg 1973;1:13-8.

14. Hamahata A, Saitou T, Ishikawa M, Beppu T, Sakurai H. Lower lip reconstruction using a combined technique of the webster and Johanson methods. Ann Plast Surg 2013;70:654-6.
15. Kuttenberger JJ, Hardt N. Results of a modified staircase technique for reconstruction of the lower lip. J Craniomaxillofac Surg 1997;25:239-44.

16. Sabattini P. Rhinoplasty and cheiloplasty operated on a single individual. Bull Sci Med (Bologna) 1838;10:387.

17. Stein SA. The classic reprint: lip repair (cheiloplasty) performed by a new method, by S.A. Stein, in: Hospitals-Meddelelser, vol. 1, 212-6, 1848. Plast Reconstr Surg 1974;53:332-7.

18. Estlander JA. A method of replacing the loss of substance in one lip in the other. Arch Klin Chir 1872;14:622-31.

19. Smith JW. The anatomical and physiologic acclimatization of tissue transplanted by the lip switch technique. Plast Reconstr Surg Transplant Bull 1960;26:40-56. 